\title{
gु \\ Fast mapping of terahertz bursting thresholds and characteristics at synchrotron light sources
}

\author{
Miriam Brosi, ${ }^{1, *}$ Johannes L. Steinmann, ${ }^{1}$ Edmund Blomley, ${ }^{2}$ Erik Bründermann, ${ }^{2}$ \\ Michele Caselle, ${ }^{3}$ Nicole Hiller, ${ }^{2, \dagger}$ Benjamin Kehrer, ${ }^{1}$ Yves-Laurent Mathis, ${ }^{2}$ \\ Michael J. Nasse, ${ }^{2}$ Lorenzo Rota, ${ }^{3}$ Manuel Schedler, ${ }^{2}$ Patrik Schönfeldt, ${ }^{2}$ \\ Marcel Schuh, ${ }^{1}$ Markus Schwarz, ${ }^{1}$ Marc Weber, ${ }^{3}$ and Anke-Susanne Müller ${ }^{1,2}$ \\ ${ }^{1}$ Laboratory for Applications of Synchrotron Radiation, Karlsruhe Institute of Technology, \\ Kaiserstraße 12, D-76131, Germany \\ ${ }^{2}$ Institute for Beam Physics and Technology, Karlsruhe Institute of Technology, \\ Hermann-von-Helmholtz-Platz, 1, D-76344 Eggenstein-Leopoldshafen, Germany \\ ${ }^{3}$ Institute for Data Processing and Electronics, Karlsruhe Institute of Technology, \\ Hermann-von-Helmholtz-Platz 1, D-76344 Eggenstein-Leopoldshafen, Germany
}

(Received 16 June 2016; published 22 November 2016)

\begin{abstract}
Dedicated optics with extremely short electron bunches enable synchrotron light sources to generate intense coherent $\mathrm{THz}$ radiation. The high degree of spatial compression in this so-called low- $\alpha_{c}$ optics entails a complex longitudinal dynamics of the electron bunches, which can be probed studying the fluctuations in the emitted terahertz radiation caused by the microbunching instability ("bursting"). This article presents a "quasi-instantaneous" method for measuring the bursting characteristics by simultaneously collecting and evaluating the information from all bunches in a multibunch fill, reducing the measurement time from hours to seconds. This speed-up allows systematic studies of the bursting characteristics for various accelerator settings within a single fill of the machine, enabling a comprehensive comparison of the measured bursting thresholds with theoretical predictions by the bunched-beam theory. This paper introduces the method and presents first results obtained at the ANKA synchrotron radiation facility.
\end{abstract}

DOI: 10.1103/PhysRevAccelBeams.19.110701

\section{INTRODUCTION}

Short intense pulses of coherent synchrotron radiation (CSR) in the terahertz (THz) frequency range are generated at synchrotron radiation facilities when electron bunches are compressed to picosecond time scales. In this operation mode the high degree of spatial compression of the optics with reduced momentum compaction factor ("low- $\alpha_{\mathrm{c}}$ optics") entails complex longitudinal dynamics of the electron bunches, leading to the so-called microbunching instability. This causes time-dependent fluctuations and strong bursts in the radiated THz intensity and is characterized by a threshold current per bunch, the so-called bursting threshold.

The bursting threshold in the generation of CSR was studied at several synchrotron light sources, e.g. ANKA [1], BESSY II [2], DIAMOND [3], MLS [4], NSLS VUV Ring [5] and SOLEIL [6]. One of the first theoretical explanations of this threshold was given by Stupakov and Heifets [7]. Later work extended the theory by considering self-interactions due to CSR and shielding effects due to the

*miriam.brosi@kit.edu

'Present address: PSI, Villigen, Switzerland.

Published by the American Physical Society under the terms of the Creative Commons Attribution 3.0 License. Further distribution of this work must maintain attribution to the author(s) and the published article's title, journal citation, and DOI. vacuum chamber, considering coasting or bunched electron beams [7-10]. First comparisons between the measured bursting threshold and theoretical models were done for the bunched-beam theory in [10,11].

This article presents a novel "quasi-instantaneous" approach to study the bursting threshold, based on highrate sampling over many turns of the radiation emitted from all electron bunches circulating in the ANKA storage ring. These so-called "snapshot measurements" drastically reduce the time necessary for mapping the bursting behavior and the bursting threshold at different machine settings. The measured bursting thresholds at these different configurations are compared to the bunched-beam theory [9], which is briefly outlined below.

\section{BUNCHED-BEAM THEORY}

For wavelengths longer than the emitting charge structure, synchrotron radiation is emitted coherently with a spectral power proportional to the number of electrons squared, the power spectrum of a single electron and a form factor, which is the modulus squared of the Fourier transform of the normalized charge distribution of the radiating electron bunch [12]. If sufficiently narrow substructures exist, CSR can be emitted at shorter wavelengths than expected from the natural bunch length. In a storage ring, above a certain threshold in the bunch current, the CSR 
impedance causes a modulation of the longitudinal phase space, which gives rise to time-evolving substructures in the longitudinal particle distribution. As a consequence of continuous changes in the form factor, this microbunching instability [7] leads to strong fluctuations in the emitted CSR power, referred to as bursting, which occur with characteristic frequencies. Formally the occurrence of substructures in the longitudinal phase space above the bursting threshold can be described by the Vlasov-FokkerPlanck equation [8], taking into account the geometry of the storage ring and accelerator parameters, like the accelerating voltage and the momentum compaction factor.

The bunched-beam theory [9] describes the influence of the vacuum chamber on a bunched electron beam. The resulting condition for the bursting threshold, $\left(S_{\mathrm{CSR}}\right)_{\mathrm{th}}$, as a function of the shielding parameter $\Pi$, in the following taken from the parallel plates model [13] for a vacuum chamber of height $2 h$, is found in [9] to be

$\left(S_{\mathrm{CSR}}\right)_{\mathrm{th}}(\Pi)=0.5+0.12 \Pi \quad$ with $\quad \Pi=\frac{\sigma_{\mathrm{z}, 0} R^{1 / 2}}{h^{3 / 2}}$.

Here the threshold is expressed in terms of the CSR strength $S_{\mathrm{CSR}}=I_{\mathrm{n}} R^{1 / 3} / \sigma_{\mathrm{z}, 0}^{4 / 3}$, with $I_{\mathrm{n}}=\sigma_{\mathrm{z}, 0} I_{\mathrm{b}} /\left(\alpha_{c} \gamma \sigma_{\delta}^{2} I_{\mathrm{A}}\right)$ the normalized bunch current, $I_{\mathrm{b}}$ the bunch current, $R$ the bending radius, $\sigma_{\mathrm{z}, 0}$ the natural bunch length, $\alpha_{\mathrm{c}}$ the momentum compaction factor, $\sigma_{\delta}$ the relative energy spread, $\gamma$ the Lorentz factor and $I_{\mathrm{A}}=4 \pi \varepsilon_{0} m_{\mathrm{e}} c^{3} / e=$ 17045 A the Alfvén current.

The above relations allow to express the bunch current at the bursting threshold

$I_{\mathrm{b}}^{\mathrm{th}}=I_{\mathrm{A}} \gamma \sigma_{\delta}^{2} \alpha_{\mathrm{c}} R^{-1 / 3} \sigma_{\mathrm{z}, 0}^{1 / 3}\left(0.5+0.12 R^{1 / 2} \sigma_{\mathrm{z}, 0} h^{-3 / 2}\right)$

as a function of parameters characterizing the longitudinal beam dynamics like the momentum compaction factor $\alpha_{\mathrm{c}}$, which is defined by the magnet optics and like the natural bunch length [14]

$\sigma_{\mathrm{z}, 0}=\frac{\alpha_{\mathrm{c}} c \sigma_{\delta}}{2 \pi f_{\mathrm{s}}} \quad$ with $\quad f_{\mathrm{s}}=\sqrt{\frac{\alpha_{\mathrm{c}} f_{\mathrm{rf}} f_{\mathrm{rev}} \sqrt{e^{2} V_{\mathrm{rf}}^{2}-U_{0}^{2}}}{E 2 \pi}}$

with the synchrotron frequency $f_{\mathrm{s}}$, the beam energy $E$, the rf frequency $f_{\text {rf }}$, the revolution frequency $f_{\text {rev }}$, the rf peak voltage $V_{\text {rf }}$ and the radiated energy per particle and revolution $U_{0}$.

\section{EXPERIMENTAL SETUP}

The core of the ANKA synchrotron radiation source of the Karlsruhe Institute of Technology, Germany, is a $110.4 \mathrm{~m}$ long electron storage ring, operating in the energy range from $0.5 \mathrm{GeV}$ to $2.5 \mathrm{GeV}$. The low- $\alpha_{\mathrm{c}}$ mode at ANKA allows the reduction of the bunch length down to a
TABLE I. Typical parameters during measurements.

\begin{tabular}{ll}
\hline \hline Electron beam energy $E / \mathrm{GeV}$ & 1.3 \\
rf frequency $f_{\text {rf }} / \mathrm{MHz}$ & 499.705 \\
Revolution frequency $f_{\text {rev }} / \mathrm{MHz}$ & 2.7157 \\
Synchrotron frequency $f_{\mathrm{s}} / \mathrm{kHz}$ & 4.5 to 13.5 \\
rf voltage $V_{\mathrm{rf}} / \mathrm{kV}$ & 500 to 1400 \\
Radiated energy $/$ particle/revolution $U_{0} / \mathrm{keV}$ & 45.5 \\
Relative energy spread $\sigma_{\delta}$ & $0.47 \times 10^{-3}$ \\
Momentum compaction factor $\alpha_{\mathrm{c}}$ & $(2.3-10) \times 10^{-4}$ \\
\hline \hline
\end{tabular}

few picoseconds with a dedicated magnet optics at $1.3 \mathrm{GeV}$ described in detail in [15]. Typical machine parameters for the presented measurements are summarized in Table I.

A state-of-the-art bunch-by-bunch feedback system [16] is used to generate custom filling patterns (e.g., a single bunch, arbitrary bunch distances, and varying bunch currents) required for the beam dynamics studies presented in this paper.

For the investigations of the microbunching instability at ANKA, a variety of fast $\mathrm{THz}$ detector systems are used in combination with fast, in-house built, high-repetition-rate data acquisition (DAQ) systems. For the bunch-by-bunch, turn-by-turn studies of the rapidly fluctuating $\mathrm{THz}$ radiation, fast detectors with different characteristics, e.g., sensitivity, or noise figures, are available at KIT. One example is an in-house developed $\mathrm{THz}$ detector system based on a YBCO sensor [17]. The investigations of this paper were performed with a broad band quasioptical Schottky diode (ACST GmbH, Hanau, Gemany) with a sensitivity in the spectral range from several $10 \mathrm{GHz}$ up to $2 \mathrm{THz}$ with the peak sensitivity around $80 \mathrm{GHz}$ [18]. With a bunch spacing at ANKA of $2 \mathrm{~ns}$, corresponding to the maximum bunch repetition rate of $500 \mathrm{MHz}$, the built-in $4 \mathrm{GHz}$ analog amplifier of the detector is fast enough to resolve the $\mathrm{THz}$ pulse of each bunch individually.

The KArlsruhe Pulse Taking and Ultrafast Readout Electronics system (KAPTURE) [19], developed at KIT, implements a memory-efficient approach to acquire the detector signal on a bunch-by-bunch basis. The signal of the fast $\mathrm{THz}$ detector is fed via high bandwidth connectors into a track-and-hold unit and a 12-bit, $500 \mathrm{MSa} / \mathrm{s}$ analogto-digital converter. KAPTURE offers up to 4 sampling channels with individual delay units, whose sampling trigger points can be adjusted in 3 ps steps each. This enables a "local sampling" of a detector signal in minimum steps of 3 ps. This local sampling depends on the chosen delays between the channels, with a maximum rate of $330 \mathrm{GSa} / \mathrm{s}$, compared to a global sampling rate of approximately $500 \mathrm{MSa} / \mathrm{s}$ in each channel [20].

For the studies detailed below, the fast $\mathrm{THz}$ detector measures the THz pulse emitted by each bunch at each turn. The DAQ system, KAPTURE, was configured to record only the amplitude of the detector response to the $\mathrm{THz}$ pulse. During a measurement period of one second, the 
amplitude of the detector pulses for each individual bunch is thus recorded for approximately 2.7 million consecutive turns. In the following, we will refer to this data as the $\mathrm{THz}$ signal of each bunch.

\section{BEAM STUDIES}

The threshold current for bursting emission of CSR depends on several (key) beam parameters, such as the momentum compaction factor and the natural bunch length [see Eq. (3)]. It can therefore provide information on the longitudinal beam dynamics. Furthermore, knowledge of the bunch currents where the microbunching instability occurs for a given set of machine parameters is also critical for experiments that rely on stable $\mathrm{THz}$ emission.

\section{A. Bursting THz radiation}

With the setup described above, the amplitude of the detector response for the individual $\mathrm{THz}$ pulse of each bunch is recorded for every turn of over 2.7 million consecutive revolutions. Such a data set contains the $\mathrm{THz}$ trace of all bunches during the measurement time of one second. The first 130 thousand turns of one such data set are displayed in Fig. 1. Each horizontal row shows the $\mathrm{THz}$ signal as a function of the turn number. A vertical column yields the $\mathrm{THz}$ signal for all bunches at a specific turn. The filling pattern for this measurement consisted of three trains with about 30 bunches each (Fig. 1). The bunch currents were measured with a time-correlated singlephoton counting setup [21].

The fluctuations in the intensity due to the bursting are clearly visible for each bunch. Bunches with different currents display a different bursting behavior, i.e., temporal evolution of a burst and its repetition rate. To measure these current-dependent changes for each bunch, a data set was taken every 10 seconds while the total beam current decreased. Combined with the measured bunch currents, these measurements allow us to map fluctuations of the

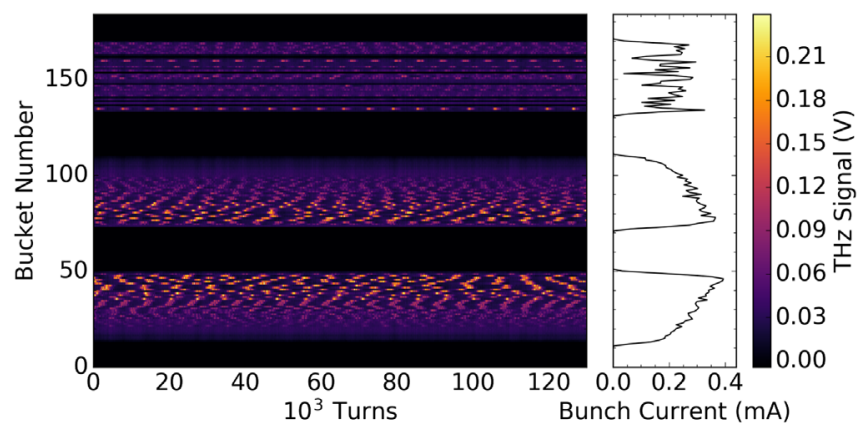

FIG. 1. The fluctuating THz signal (color coded) of each of the 184 rf-buckets is shown for the first 130 thousand consecutive turns, out of the total recorded of 2.7 million in one second. The bursting behavior differs for bunches with different currents. On the right-hand side the filling pattern, consisting of three trains, is indicated by the bunch current (adapted from [22]).

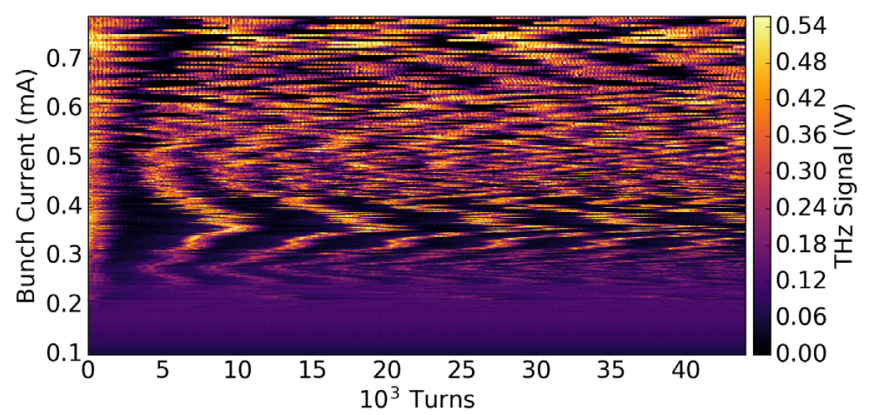

FIG. 2. THz signal (color coded) of one bunch as a function of turn number during the decrease of the bunch current. Both the temporal evolution and the repetition rate of an outburst show a strong dependence on bunch current. For better visibility the $\mathrm{THz}$ signals were shifted in time so that the first bursts are aligned.

intensity emitted by each bunch as a function of the turn number $\left(T_{\text {rev }}=368 \mathrm{~ns}\right.$ for ANKA) and of the decreasing bunch current (time scale typically between one and several hours). An improved measurement technique, which reduces the measurement time by combining all bunches, is described in the next section.

In Fig. 2 the $\mathrm{THz}$ signal of one individual bunch is displayed as a function of the decreasing bunch current, showing changes in the bursting behavior as well as the onset of bursting in the detection range of the detector system used for this study, in this case, at $0.2 \mathrm{~mA}$.

\section{B. Fast mapping technique}

The slow measurement technique described in the previous section collects the $\mathrm{THz}$ signal traces of all 184 rf-buckets for one second, during which time the bunch currents stay approximately constant. This way, a study of current dependent effects is usually achieved by repeating those one-second-measurements of $\mathrm{THz}$ signal traces for changing beam currents. To avoid possible influences from continued beam injection, or in the absence of a full energy injection, the measurement series are performed while the beam current slowly decays due to a limited beam lifetime over a time span of several hours.

The fast mapping technique (the so-called "snapshot" measurement), enabled by the unique combination of fast $\mathrm{THz}$ detectors with high-data-throughput DAQ systems, drastically reduces the time required to cover the full bunch current range of interest: instead of following one single bunch during an hour-long beam current decrease, the technique makes use of the quasisimultaneous acquisition of all bunches, for this purpose filled to cover the full current range of interest, to achieve the same result in one second. In the following it will be assumed that the multibunch effects on the measured thresholds are small and will be included as part of the error propagation on the detected bursting thresholds. The special, tailored filling pattern required for the measurements is achieved with the help of a bunch-by-bunch feedback system [16]. 


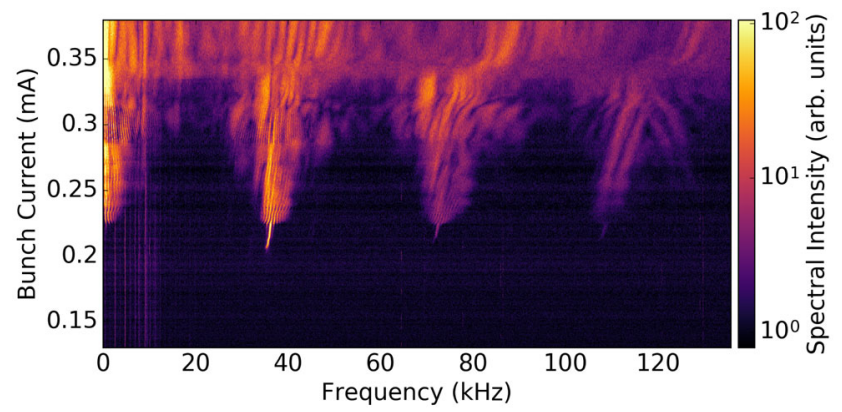

(a)

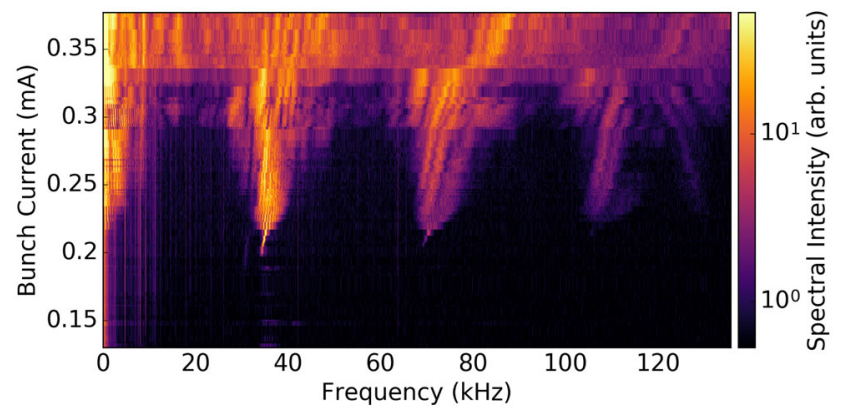

(b)

FIG. 3. The spectrogram shown in (a) was obtained from measurements of a single bunch for slowly changing beam current lasting two and a half hours, while the spectrogram in (b) was obtained from a snapshot measurement lasting just one second by using the FFT of THz signals similar to those shown in Fig. 1. For the snapshot measurement the bunch current resolution is limited by the number of bunches and their current distribution. Compared to this, the standard measurement during a slow current decrease results in a higher current resolution. Despite the limited resolution of the snapshot spectrogram, the dominant bursting frequencies and the thresholds between different bursting regimes are clearly visible.

With such a filling pattern, a snapshot measurement of one data set within one second is sufficient to analyze the bursting behavior at different bunch currents and to determine the bursting threshold corresponding to the present machine settings. Measurements, which previously lasted typically up to 5 hours, depending on the lifetime of the electron beam, now only require one second or less.

\section{Mapping the bursting behavior}

The snapshot measurements offer the opportunity to acquire fast, comprehensive maps of the microbunching instability in dependence of various accelerator parameters. To visualize the change in bursting periodicity as a function of the bunch current, the Fourier transform of the $\mathrm{THz}$ signal is displayed for the investigated bunch current range in a spectrogram (see Fig. 3). Bunch currents that mark a change in the fluctuation of the $\mathrm{THz}$ signal and therefore in the driving microbunching instability are easily accessible in the visualization [23]. With the quasisimultaneous acquisition of bunches covering the full bunch current range, a spectrogram can now be created from just one data set.

The resulting spectrogram has a limited resolution on the current axis, due to the limited number of bunches and hence current bins, as shown in Fig. 3(b) in comparison to the spectrogram obtained from measurements of a single bunch for different bunch currents shown in Fig. 3(a). However, the different dominant frequencies and regions are clearly visible and give an overview of the bursting behavior for these accelerator settings. A comparison between the snapshot spectrogram and a spectrogram taken during a standard current decay of one bunch at the same accelerator settings (Fig. 3) shows the excellent agreement of the two methods and that the dominant structures can easily be reproduced by the time-saving snapshot measurement. This supports the assumption that the bursting behavior of the microbunching instability in these measurements is dominated by a single bunch effect.

\section{Fast threshold determination}

To extract the bursting threshold from a snapshot measurement, the standard deviation of the $\mathrm{THz}$ signal of each bunch is calculated and displayed as a function of the momentary current of the bunch in question (Fig. 4). The bursting threshold is immediately visible as a kink in the standard deviation, because the $\mathrm{THz}$ signal is stable at currents below the threshold. Above the instability threshold, when the $\mathrm{THz}$ signal starts to fluctuate over the turns, the value of the standard deviation increases.

Figure 4 shows that the $\mathrm{THz}$ signals of bunches below the threshold of $0.2 \mathrm{~mA}$ show hardly any fluctuations compared to the signal of bunches with higher current.

\section{Bursting threshold and beam optics}

The bursting threshold $I_{\mathrm{b}}^{\text {th }}$ depends on the momentum compaction factor $\alpha_{\mathrm{c}}$ and the natural bunch length $\sigma_{\mathrm{z}, 0}$ [Eq. (2)]. The natural bunch length $\sigma_{z, 0}$ and therefore the threshold can be influenced by the momentum compaction

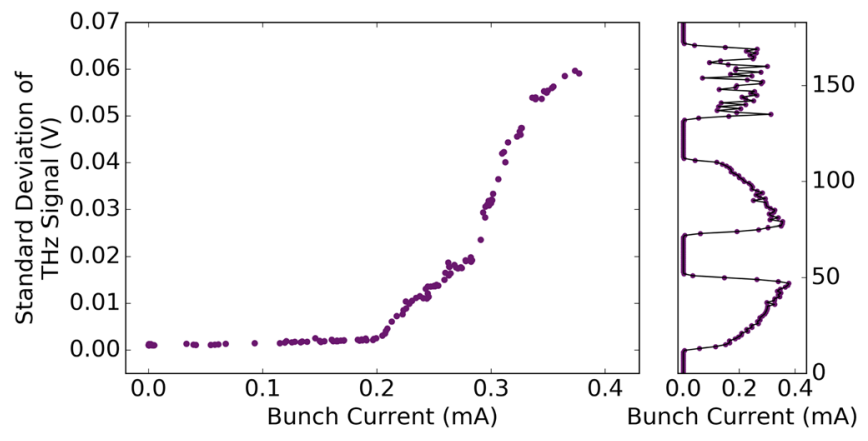

FIG. 4. Standard deviation of the THz signal of each bunch as a function of the corresponding bunch current, revealing the onset of bursting emission ("bursting threshold") at $0.2 \mathrm{~mA}$. The individual bunch currents (shown on the right) were patterned to equally sample the whole bunch current range (adapted from [22]). 


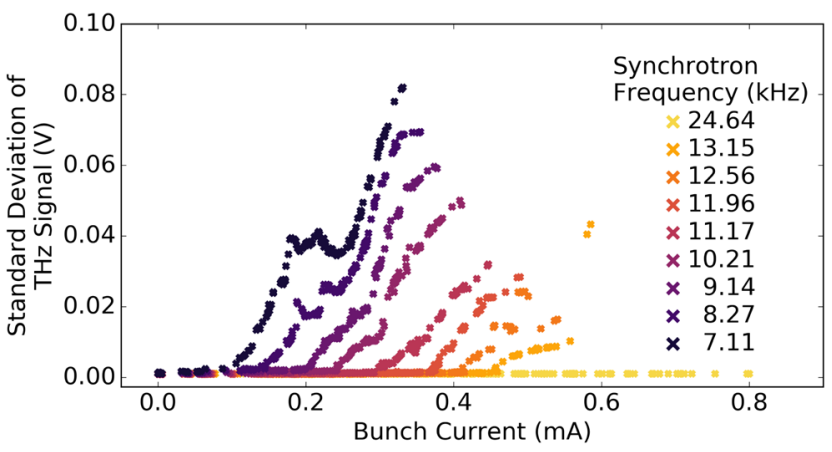

FIG. 5. Standard deviation of the THz signal as a function of the bunch current for a constant rf-voltage of $1047 \mathrm{kV}$ and different settings of the magnet optics, resulting in different synchrotron frequencies and hence different bursting thresholds (adapted from [24]).

factor (by changing the magnet optics) and by the $\mathrm{rf}$ voltage [Eq. (3)].

To study the dependency from the magnet optics, snapshot measurements were used to determine the bursting threshold for different settings of the magnet optics at a fixed rf-voltage. The different optic settings resulted in different values of the momentum compaction factor and thus of the natural bunch length. According to Eq. (3) these changes will lead to a difference in the synchrotron frequency, which will be measured to monitor the stepwise changes in the magnet optics. In the following, the stepwise change of the magnet optics will be referred to as "magnet sweep." At each step a snapshot measurement was taken.
Figure 5 shows the standard deviation of the intensity of the $\mathrm{THz}$ signal as a function of the bunch current for several steps of the sweep. With decreasing synchrotron frequency $f_{\mathrm{s}}$ a change in the threshold, i.e., the onset of the fluctuations, is visible.

Displaying the bursting threshold in dependence of the measured synchrotron frequency $f_{\mathrm{s}}$ for each step of the magnet sweep shows the correlation even more clearly (Fig. 6). The decrease of the threshold with decreasing synchrotron frequency matches the expected behavior. For a fixed acceleration voltage the synchrotron frequency decreases for a lower momentum compaction factor, which leads to a shorter bunch length. For a shorter bunch length the critical charge density above which the microbunching instability occurs is reached at a lower current. For a fixed rf-voltage, a lower synchrotron frequency corresponds to a lower bursting threshold (Fig. 6).

The error on the threshold contains the uncertainties of the threshold detection algorithm, the measured bunch current and the error expected by the spread of the threshold due to multibunch effects [22].

\section{Bursting threshold, beam optics, and rf-voltage}

In a second measurement the rf-voltage was changed stepwise together with the magnet optics, in the following referred to as "combined sweep." Figure 6 shows the measured bursting thresholds as a function of the measured synchrotron frequency. For a rf-voltage of $1047 \mathrm{kV}$ the thresholds are in good agreement with the thresholds seen

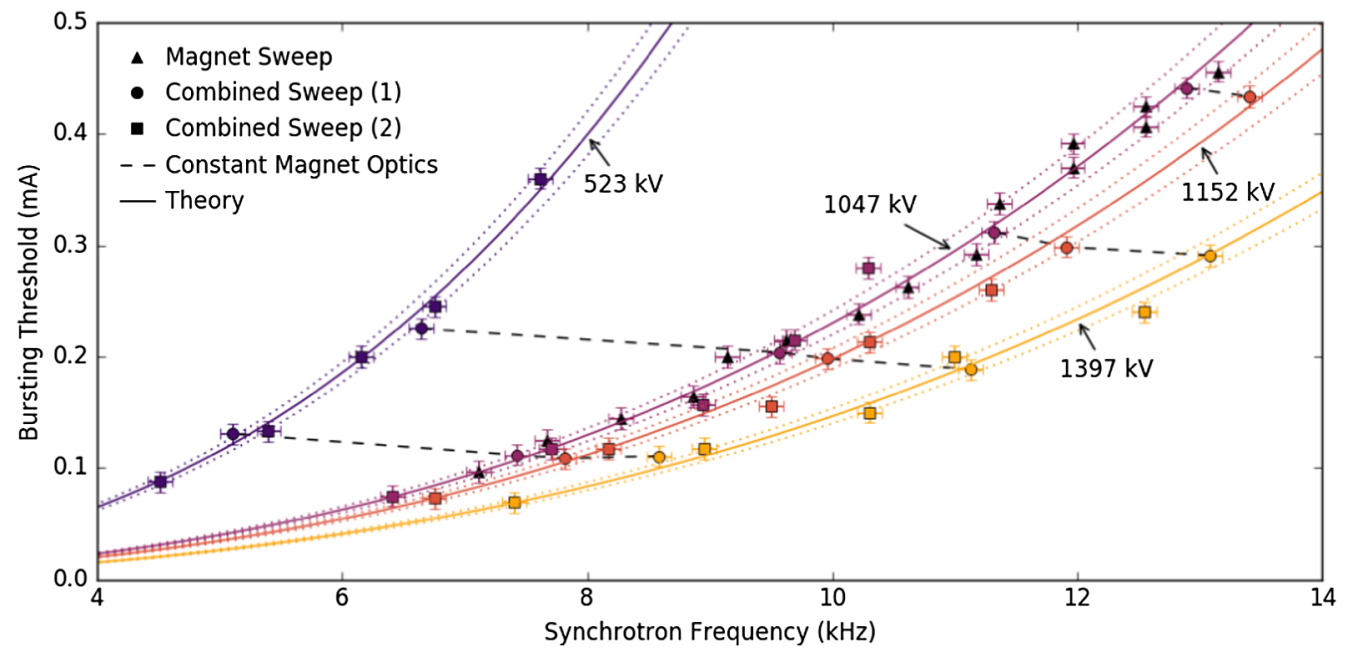

FIG. 6. Measured bursting thresholds from three sets of measurements as a function of the synchrotron frequency. Here "combined sweep (2)" refers to the same measurements taken at 11 months apart from "combined sweep (1)." For "combined sweep (1)," measurement with the same magnet optics are connected by dashed lines. The different colors indicate the different acceleration voltages. The horizontal error bars indicate the systematic error on the measured synchrotron frequency. The vertical error bars include the error of the algorithm for the automated threshold detection, the bunch current measurement as well as the expected spread of the threshold due to multibunch effects. The solid lines show the theoretical expectation according to Eq. (2) from the bunched-beam theory. The dotted lines indicate the one standard deviation uncertainties on the theoretical predictions. Within uncertainties all measurements agree with the bunched-beam theory. 
in the magnet sweep study (Sec. IV B 3), where the same voltage was used.

As described above, a bunch length reduction due to a change of the magnet optics that leads to a lower momentum compaction factor, decreases the threshold. Furthermore, for a fixed momentum compaction factor, a shortening of the bunch by an increase in the rf-voltage which is accompanied by an increase of the synchrotron frequency [Eq. (3)] also results in a decrease of the threshold. For changes of the rf-voltage, the relative change of the threshold with the synchrotron frequency is smaller than the change due to changes of the momentum compaction factor (Fig. 6).

\section{COMPARISON WITH BUNCHED-BEAM THEORY}

In the following, the measured bursting thresholds are compared to absolute predictions of the bunched-beam theory.

For this comparison the synchrotron frequency $f_{\mathrm{s}}$ as well as the accelerating voltage were determined for each setting during the measurements. As it was not possible to measure the momentum compaction factor for each change-step of the magnet optics during the presented measurements, the compact Compton backscattering setup at ANKA [25] was used to determine the momentum compaction factor for several different magnet optics in independent measurements [26]. This allowed the extraction of a voltage calibration factor, which relates the acceleration voltage $V_{\text {rf }}$ seen by the electrons to the set values of the cavities. With this factor we determined the momentum compaction factor from the synchrotron frequency and the rf voltage. The knowledge of these parameters combined with the machine parameters from Table I is then used to calculate the theoretically predicted bursting threshold according to Eq. (4):

$$
\begin{aligned}
I_{\mathrm{b}}^{\mathrm{th}}= & \frac{I_{\mathrm{A}} \gamma \sigma_{\delta}^{7 / 3} 2 \pi c^{1 / 3} E^{4 / 3} f_{\mathrm{s}}^{7 / 3}}{R^{1 / 3} f_{\mathrm{rf}}^{4 / 3} f_{\mathrm{rev}}^{4 / 3}\left(\sqrt{e^{2} V_{\mathrm{rf}}^{2}-U_{0}^{2}}\right)^{4 / 3}} \\
& \times\left(0.5+0.12 \frac{R^{1 / 2} E c \sigma_{\delta} f_{s}}{h^{3 / 2} f_{\mathrm{rf}} f_{\mathrm{rev}} \sqrt{e^{2} V_{\mathrm{rf}}^{2}-U_{0}^{2}}}\right)
\end{aligned}
$$

which was derived by applying Eq. (3) for the natural bunch length and the momentum compaction factor to Eq. (2).

Figure 6 shows the measured bursting thresholds of three different sets of measurements, the magnet sweep and two combined sweeps (taken 11 months apart) as a function of the synchrotron frequency. The theoretical prediction according to Eq. (2) for the four different acceleration voltages is shown, and the one standard deviation uncertainty on the theoretical values, calculated from the errors on the measured input parameters used in Eqs. (3) and (2), is indicated. Within the uncertainties, the measured thresholds agree extremely well with the theoretical values, derived from the model as detailed in Sec. II.

\section{CONCLUSION}

Fast snapshot measurements, enabled by the unique combination of fast $\mathrm{THz}$ detectors with the high-datathroughput KAPTURE DAQ system, drastically speed up time consuming surveys of the microbunching instability for different accelerator settings. The quasisimultaneous acquisition of $\mathrm{THz}$ signals from all bunches allows the determination of the bursting threshold and the calculation of a spectrogram. This spectrogram shows the characteristic bursting frequencies from a single data set covering one second of beam time, compared to the previously used methods requiring several hours. This speed-up by about 4 orders of magnitude permits systematic studies of the bursting threshold for a large set of machine parameters within the same fill. The reproducibility of this method is confirmed by the consistency of the results from two similar measurements performed 11 months apart. With measurements of only three different fills, it was possible to gather sufficient data to demonstrate that the absolute predictions of the bunched-beam theory for the bursting threshold are in excellent agreement with the measurements.

\section{ACKNOWLEDGMENTS}

The authors would like to thank the infrared group at ANKA and in particular M. Süpfle for his support during the beam times at the IR1 and IR2 beam lines as well as Cheng Chang for the momentum compaction factor data obtained by Compton backscattering measurements (CBS) and Heinz-Wilhelm Hübers and Heiko Richter (DLR, Berlin) for providing the necessary $\mathrm{CO}_{2}$ laser for the $\mathrm{CBS}$ measurements. Further, the authors would like to thank the ANKA $\mathrm{THz}$ group for inspiring discussions. This work has been supported by the German Federal Ministry of Education and Research (Grant No. 05K13VKA), and the Helmholtz Association (Contract No. VH-NG-320). Miriam Brosi acknowledges the financial support of the Helmholtz International Research School for Teratronics (HIRST).

[1] A.-S. Müller, Accelerator-based sources of infrared and terahertz radiation, Rev. Accel. Sci. Techol. 03, 165 (2010).

[2] M. Abo-Bakr, J. Feikes, K. Holldack, P. Kuske, and G. Wustefeld, Coherent emission of synchrotron radiation and longitudinal instabilities, in Proceedings of the 2003 Particle Accelerator Conference, Portland, OR (IEEE, New York, 2003), p. 3023.

[3] W. Shields, R. Bartolini, G. Boorman, P. Karataev, A. Lyapin, J. Puntree, and G. Rehm, Microbunch instability observations from a $\mathrm{THz}$ detector at diamond light source, J. Phys. Conf. Ser. 357, 012037 (2012).

[4] G. Wüstefeld, J. Feikes, M. Hartrott, M. Ries, A. Hoehl, R. Klein, R. Müller, A. Serdyukov, and G. Ulm, Coherent THz 
Measurements at the Metrology Light Source, in Proceedings of the International Particle Accelerator Conference, Kyoto, Japan, 2010 (ICR, Kyoto, 2010), p. 2508.

[5] G. Carr, S. Kramer, J. Murphy, J. LaVeign, R. Lobo, D. Reitze, and D. Tanner, Investigation of coherent emission from the NSLS VUV ring, in Proceedings of the 18th Particle Accelerator Conference, New York, 1999 (IEEE, New York, 1999), p. 134.

[6] C. Evain, J. Barros, A. Loulergue, M. A. Tordeux, R. Nagaoka, M. Labat, L. Cassinari, G. Creff, L. Manceron, J. B. Brubach, P. Roy, and M. E. Couprie, Spatio-temporal dynamics of relativistic electron bunches during the microbunching instability in storage rings, Europhys. Lett. 98, 40006 (2012).

[7] G. Stupakov and S. Heifets, Beam instability and microbunching due to coherent synchrotron radiation, Phys. Rev. Accel. Beams 5, 054402 (2002).

[8] M. Venturini and R. Warnock, Bursts of Coherent Synchrotron Radiation in Electron Storage Rings: A Dynamical Model, Phys. Rev. Lett. 89, 224802 (2002).

[9] K. L. F. Bane, Y. Cai, and G. Stupakov, Threshold studies of the microwave instability in electron storage rings, Phys. Rev. Accel. Beams 13, 104402 (2010).

[10] Y. Cai, Theory of Microwave Instability and Coherent Synchrotron Radiation in Electron Storage Rings, in Proceedings of the 2nd International Particle Accelerator Conference, San Sebastián, Spain (EPS-AG, Spain, 2011), p. 3774.

[11] M. Ries, J. Feikes, P. Schmid, G. Wüstefeld, and A. Hoehl, THz Bursting Thresholds Measured at the Metrology Light Source, in Proceedings of the 3rd International Particle Accelerator Conference, New Orleans, LA, 2012 (IEEE, Piscataway, NJ, 2012), p. 3030.

[12] A.-S. Müller et al., Experimental Aspects of CSR in the ANKA Storage Ring, in Beam Dynamics Newsletter, Vol. 57, edited by Y. Cai and W. Chou (International Committee for Future Accelerators, 2012), http://www-bd .fnal.gov/icfabd/Newsletter57.pdf.

[13] J. Murphy, S. Krinsky, and R. Gluckstern, Longitudinal wakefield for an electron moving on a circular orbit, Part. Accel. 57, 9 (1997).

[14] H. Wiedemann, Handbook of Accelerator Physics and Engineering, 2nd ed. (World Scientific, Singapore, 2013), Chap. 3.1.4, p. 220.

[15] A.-S. Müller, I. Birkel, B. Gasharova, E. Huttel, R. Kubat, Y.-L. Mathis, D. Moss, W. Mexner, R. Rossmanith, M. Wuensch, P. Wesolowski, F. Pèrez, M. Pont, and C. J. Hirschmugl, Far Infrared Coherent Synchrotron Edge Radiation at ANKA, in Proceedings of the 21st Particle Accelerator Conference, Knoxville, TN, 2005 (IEEE, Piscataway, NJ, 2005).

[16] E. Hertle, E. Huttel, A.-S. Müller, N. Smale, D. Teytelman, and M. Höner, First Results of the new bunch-by-bunch feedback system at ANKA, in Proceedings of 2014
International Particle Accelerator Conference, Dresden, Germany (JACoW, Geneva, 2014), p. 1739.

[17] J. Raasch et al., Investigation of the Electrical Field Sensitivity of Sub- $\mu \mathrm{m} \mathrm{Y}-\mathrm{Ba}-\mathrm{Cu}-\mathrm{O}$ Detectors, IEEE Trans. Appl. Supercond. 25, 1 (2015).

[18] Advanced Compound Semiconductor Technologies (ACST) GmbH, UWB Quasi-optical Detectors up to $2 \mathrm{THz}$, Flyer.

[19] C. Caselle, M. Brosi, S. Chilingaryan, T. Dritschler, E. Hertle, V. Judin, A. Kopmann, A.-S. Müller, J. Raasch, M. Schleicher, M. Siegel, N. Smale, J. Steinmann, M. Vogelgesang, M. Weber, and S. Wuensch, Commissioning of an Ultra-fast Data Acquisition System for Coherent Synchrotron Radiation Detection, in Proceedings of 2014 International Particle Accelerator Conference, Dresden, Germany (JACoW, Geneva, 2014), p. 3497.

[20] M. Caselle, M. Balzer, S. Chilingaryan, M. Hofherr, V. Judin, A. Kopmann, N. J. Smale, P. Thoma, S. Wuensch, A.-S. Müller, M. Siegel, and M. Weber, An ultra-fast data acquisition system for coherent synchrotron radiation with terahertz detectors, J. Instrum. 9, C01024 (2014).

[21] B. Kehrer, A. Borysenko, E. Hertle, N. Hiller, M. Holz, A. Müller, P. Schönfeldt, and P. Schütze, Visible Light Diagnostics at the ANKA Storage Ring, in Proceedings of 2015 International Particle Accelerator Conference, Richmond, VA (JACoW, Geneva, 2015), p. 866.

[22] M. Brosi, M. Caselle, E. Hertle, N. Hiller, A. Kopmann, A.-S. Müller, P. Schönfeldt, M. Schwarz, J. L. Steinmann, and M. Weber, Online Studies of THz-Radiation in the Bursting Regime at ANKA, in Proceedings of 2015 International Particle Accelerator Conference, Richmond, VA (JACoW, Geneva, 2015), p. 882.

[23] M. Schwarz, V. Judin, A.-S. Müller, and M. Klein, Bursting Patterns of Coherent Synchrotron Radiation in the ANKA Storage Ring, in Proceedings of the 4th International Particle Accelerator Conference, IPAC2013, Shanghai, China, 2013 (JACoW, Shanghai, China, 2013), p. 2516.

[24] V. Judin, M. Brosi, C. Caselle, E. Hertle, N. Hiller, A. Kopmann, A.-S. Müller, M. Schuh, N. Smale, J. Steinmann, and M. Weber, Studies of Bursting CSR in Multi-bunch Operation at the ANKA Storage Ring, in Proceedings of 2014 International Particle Accelerator Conference, Dresden, Germany (JACoW, Geneva, 2014), p. 225 .

[25] C. Chang, E. Bründermann, E. Hertle, N. Hiller, E. Huttel, A.-S. Müller, M. J. Nasse, M. Schuh, J. L. Steinmann, H.-W. Hübers, and H. Richter, First Results of Energy Measurements with a Compact Compton Backscattering Setup at ANKA, in Proceedings of 2015 International Particle Accelerator Conference, Richmond, VA (JACoW, Geneva, 2015), p. 876.

[26] C. Chang, Ph.D. thesis, KIT, Karlsruhe, Germany, 2016. 